\title{
Research on the Allocation of Urban and Rural Public Service Facilities Based on Life Circle Theory-A Case Study of Honghu City, Hubei Province
}

\author{
Tianlin Su \\ College of Architecture, Southwest Minzu University, Chengdu, China \\ Email: 962761697@qq.com
}

How to cite this paper: Su, T.L. (2021) Research on the Allocation of Urban and Rural Public Service Facilities Based on Life Circle Theory-A Case Study of Honghu City, Hubei Province. Open Access Library Journal, 8: e7852.

https://doi.org/10.4236/oalib.1107852

Received: August 12, 2021

Accepted: August 27, 2021

Published: August 30, 2021

Copyright $\odot 2021$ by author(s) and Open Access Library Inc.

This work is licensed under the Creative Commons Attribution International License (CC BY 4.0).

http://creativecommons.org/licenses/by/4.0/

\begin{abstract}
With the continuous improvement of living standard, the improvement of public service facilities is an essential topic in urban and rural construction. Public service facilities are a bridge to promote the equalization of public service facilities at an early date, to create a better living environment and to ensure social equity. This paper studies the allocation of public service facilities in Honghu City, applies the theory of living circles to the allocation of urban and rural public service facilities, and divides Honghu City into urban living circles and community living circles based on the specific conditions of Honghu City. Furthermore, the community life circle is refined into a 15 -minute community life circle, a 10-minute community life circle, and a 5-minute community life circle. Based on the theory of living circle, this paper studies the allocation of urban and rural public service facilities in Honghu City, and puts forward the guidance of urban and rural public service facilities allocation based on the theory of living circle, which is conducive to creating a urban and rural environment with perfect facilities, reasonable layout and meeting people's needs, At the same time, it also provides some reference significance for the allocation of urban and rural public service facilities.
\end{abstract}

\section{Subject Areas \\ Urban Planning}

\section{Keywords}

Public Service Facilities, Life Circle Theory, Town Life Circle, Community Life Circle 


\section{Introduction}

Since the reform and opening up, with the improvement of living standards in China, people's ideas have also changed a lot. In the past, people have reflected on the concept of "seeing things without people". Urban planning is for the people; the city is the people's city; urban planning should start from people, people-oriented. To create a more suitable city for people to live in, the city should make people's life more beautiful. The development of the city cannot be separated from the residents' demand for public facilities. With the continuous upgrading of the residents' demand for public facilities, the equalization of public facilities is an important condition for the development of the city. It is also an important topic studied by scholars today. Generally speaking, the city's public service facilities are figured according to the thousand-person index. With the development of the times, residential areas planning standards are also constantly improving in China. It also puts forward new requirements for the configuration of public service facilities in urban residential areas [1]. The deployment of public service facilities in the new design standards encourages the adaptation of public service facilities to specific needs. Under this background, the layout of urban and rural public service facilities cannot be applied to the allocation of public service facilities according to the previous requirements and new standards. It is of great practical significance to realize the equalization of public service facilities and to provide some reference for the layout of public service facilities in the future.

\section{Conceptual Analysis}

The concept of life circle was first put forward in Japan's comprehensive development plan, and then popularized in Korea and Taiwan. Life Circle refers to the time and space range formed by residents when they travel (including shopping, commuting and leisure) to meet their production and living needs [2]. Many scholars in our country have also defined the concept of life circle. For example, Yuan Jiadong and others think that the daily life circle is a space for residents to carry out ordinary activities and has certain functions at the same time. Sun Defang believes that in order to satisfy the regional system of survival, development and communication, people in the life circle can satisfy many kinds of behavior activities, such as people's residence, work, medical treatment and so on. Liu Yungang and others pointed out that the circle of life is a circle formed by residents connecting the central area with the surrounding area. Because of the different definition of the concept of life circle, the application of life circle theory also has various forms [3]. Chen Qinghui and others applied the concept of "life circle" to the quality evaluation of urban living environment. Zhu Chasong and others constructed the public service facility configuration system based on different levels of life circle [4]. The theory of life circle takes human needs as the starting point and combines the theory of life circle with the layout of urban and rural public service facilities through the application of life circle 
theory, which can better realize the equalization of public service facilities, improve the utilization rate of public service facilities, and realize the rational allocation of public service facilities resources.

\section{Research on Public Service Facilities Allocation in Honghu Lake Based on Life Circle Theory}

\subsection{Regional Overview of the Study}

Honghu City is located in the central and southern part of Hubei Province, belonging to Jingzhou City, named after the largest lake Honghu. For Honghu City the total area is 2519 square kilometers, as of 2019, Honghu registered population of 913,200 people. In recent years, because Honghu is about 150 kilometers from Wuhan, close to Wuhan, convenient transportation and good location, it has been approved as the "observer". With the full opening of Honghu to supervision highway in September 2019, the passage time from Honghu to Wuhan is greatly shortened, and the communication between Honghu and Wuhan is more convenient. Honghu is also located as a satellite city in Wuhan city circle. Honghu is mainly to develop tourism and the sale of aquatic products. Honghu aquatic products are rich in resources and are the pillar industries of Honghu City. The public service facilities in Honghu City are mainly residential, leisure and entertainment, business office and cultural construction. The construction of public service facilities in Honghu City has been quite effective in recent years. With the renovation and renovation of Jiangtan Park and Neijing River Long Street, the level of public service facilities has been greatly improved. However, the allocation of public service facilities in Honghu City is incomplete and the distribution of public service facilities is unreasonable. Public service facilities are mainly concentrated in the area of Jiangtan Park and Longjie. The public service facilities in the newly built development zone are not enough. This paper hopes to solve the problem of uneven distribution and insufficient allocation of public service facilities in Honghu City by studying the allocation of public service facilities in Honghu City on the basis of the theory of life circle.

\subsection{Status of Public Service Facilities in Honghu City}

The development of Honghu City is relatively backward, and the present situation of public service facilities is general. This paper mainly studies the allocation of public service facilities in Honghu City from the three public welfare service facilities of education, medical treatment and cultural facilities, which are embodied in the following aspects. Education and sports have developed steadily. As of 2019, Honghu City in educational facilities, the city's total of 90 schools, including primary schools, general secondary schools, secondary professional schools and special schools 48, 40,1 and 1. Honghu City students have a total of about 77,900 people. Primary schools, general secondary schools and secondary specialized schools were $44,400,30,400$ and 2800 respectively. The total number of 76 kindergartens is about 22,200 . In cultural facilities, there are four theatres and theatres in Honghu, The library has 88,100 books. In medical facilities, 
Honghu has 29 hospitals and hospitals as of 2019, 2941 technicians from hospitals, hospitals, 105 more than the previous year, 3316 beds, an increase of 274 over the previous year. The infrastructure level of Honghu City is lower than that of the other counties in Hubei Province. It is of great practical significance to perfect the public service facilities of Honghu City, realize the equalization of public service facilities, and improve the quality of life of Honghu citizens.

\subsection{The Division of Living Circle in Honghu City}

According to the division principle of life circle and the specific situation of Honghu city, the life circle of Honghu city is divided into urban life circle and community Life circle to build. Urban life circle is based on the whole county as the standard to build, serving the whole Honghu City. In the urban life circle, we should ensure that all kinds of public service facilities are fully equipped to meet the requirements of leisure, entertainment, education, medical treatment and so on. At the same time, we should pay attention to the coordination of the allocation of public service facilities between the urban life circle and the community life circle to ensure the maximum utilization of public service facilities.

Community life circle is constructed according to the service radius of 800 1000 meters. With the proposal of 5 minutes life circle, 10 minutes life circle and 15 minutes life circle, the delineation of community life circle in Honghu city is combined with the new standard, and the community life circle in Honghu city is defined according to the planning of 15 minutes community life circle, 10 minutes community life circle and 5 minutes community life circle [5]. Within the 5-minute community life circle, people can enjoy public service facilities such as sports and fitness venues, cultural activity stations, kindergartens, etc. Within the 10-minute community life circle, people can enjoy public service facilities such as primary schools, health stations, multi-functional sports venues. Within the 15-minute community life circle, people can enjoy public services such as culture, education and health within a 15 -minute walk [6]. So that residents can enjoy one-stop service in the community life circle, meet people's basic living needs, and construct a living space and community life circle with intensive and efficient land use, perfect facilities, comfortable life and rich business form. It is more convenient and beautiful for people to live. Secondly, the service radius of the service facilities in the 15-minute community life circle is about 1 $\mathrm{km}$, which can serve the population of 3 - 5 million people. Combined with the specific situation of Honghu City, the life-type road is constructed to meet the daily life and leisure and entertainment services of residents.

\section{Guide of Honghu Public Service Facilities Based on the Theory of Living Circle}

With the continuous development of society, the living standard of the residents has been greatly improved, and people's lives are becoming much richer. Living more and more pay attention to healthy green, square dance and fitness activities, residents of community service requirements are becoming higher and 
higher. To realize the equalization of public service facilities, to enrich the residents' entertainment, sports and cultural activities, and to improve the quality of life of residents, and to create a better living environment for residents. Now from the four aspects of public service facilities allocation principle, configuration thinking, configuration system and configuration guidance, optimize the allocation of public service facilities in Honghu City.

\subsection{Configuration Principles}

The allocation of public service facilities should fully meet the needs of people, so the allocation of public service facilities should fully follow the characteristics of people-oriented and spatial balance, and the public service facilities are open, people can freely choose public service facilities. Therefore, we should pay attention to the rational allocation of resources and avoid the repeated allocation of public service facilities. 2020 is an extraordinary year, with the global outbreak of new coronary pneumonia, the world's economic life has been seriously affected, the outbreak of new coronary pneumonia has a certain impact on the allocation of public service facilities, In the allocation of public service facilities, we should consider the principle of health, consider the allocation of public service facilities for daily health emergency, and improve the allocation of public service implementation in development.

\subsection{Configuration Ideas}

By using reasonable and scientific methods to divide the life circle of Honghu City, we can combine the wishes of local residents and understand the ideas of the masses by questionnaire. After dividing the living circle, the public service facilities in Honghu City are allocated reasonably. Configure public service facilities at all levels according to the established allocation standards of public service facilities. At the same time, we should be good at using the ARCGIS analysis function, study the layout of public service facilities in each circle, and remove the overlapping or conflicting public service facilities in the community life circle and the urban life circle. Ensure the rationality of public service facilities and avoid waste. According to the population situation of Honghu City, according to the place where the population of Honghu City is concentrated, the center of public service facilities to be allocated at all levels of life circle is found out. Then according to the layout of the public service facilities in Honghu City and the distribution plan of the infrastructure of each town and village in Honghu City, the public service facilities suitable for the specific situation of Honghu City are allocated. Community life circle is a public space, sometimes including multiple communities, residents of multiple communities to enjoy these supporting public service facilities, so in the planning and design of good land, Ensure that public service facilities can be reasonably configured.

\subsection{Configuration Boot}

The allocation of public service facilities must be considered in the light of the 
travel situation of local residents. In order to make the allocation of public service facilities in Honghu City more perfect, more scientific and reasonable, and to make the allocation of urban and rural public service facilities more people-oriented and humanized, it is necessary to take into account the daily travel of residents. At the same time, the scale effect of public service facilities and the convenience of public service authorities should be considered. The allocation of public service facilities in Honghu community life circle is based on the three types of community life circle put forward in 2018 "Urban Residential District Planning and Design Standard", and the reasonable division of Honghu life circle is carried out according to the specific conditions of Honghu city. Configure the corresponding public service facilities for different levels of life circle. In the urban life circle to meet the educational, medical, cultural and sports requirements of public service facilities, the community life circle of Honghu City is divided into 15 minutes community life circle, 10 minutes community life circle and 5 minutes community life circle. It is configured according to different levels of community life circle, such as public service facilities such as kindergarten, cultural activity station, sports fitness site and so on in 5 minutes community life circle, and public service facilities such as primary school, health station and multi-function sports site are added in 10 minutes community life circle. In the 15-minute community life circle, the addition of secondary schools, community sports centers and other public service facilities to ensure that the 15-minute community life circle can meet the education, medical and other activities. The allocation of public service facilities combined with the specific conditions of different circles is conducive to the equalization of public service facilities.

\section{Conclusion}

With the development of society, the development of China today not only focuses on the improvement of economic level, but also on the improvement of social and cultural connotation. Urban planning should solve urban problems from the perspective of human beings, and urban planning should be social. Public service facilities are the bridge between human and natural environment and the facilities for people in urban and rural areas. Therefore, the allocation of public service facilities should take full account of people's needs. The way people travel, travel preferences and travel time in urban and rural areas affect the use and effect of public service facilities. We should consider many reasons when configuring public service facilities, and make reasonable allocation of public service facilities in each region from the perspective of region. Based on the theory of life circle, this paper studies the allocation of public service facilities in Honghu City, Hubei Province, and provides some methods and theories for the allocation of public service facilities in other cities and counties, which has certain reference significance. Of course, there are some limitations in this paper. When dividing the living circle of Honghu City, the basis of the division 
is not clear enough, the research on the types of public service facilities is not perfect, and the location of Honghu City is not subdivided. In the future research, we can use some quantitative methods to configure the public service facilities, such as using the TIN analysis model in the ArcGIS to divide the time distance of each region more reasonably and scientifically, so that the public service facilities in each circle can be optimized.

\section{Conflicts of Interest}

The author declares no conflicts of interest.

\section{References}

[1] Wang, Y.T., He, P.G. and Fu, W. (2019) Exploration of Urban Block Space Environment Construction Strategy Based on Quality Orientation. 2019 Urban Development and Planning Proceedings, China Urban Science Research Association, Zhengzhou Municipal People's Government, Henan Provincial Department of Natural Resources, Henan Provincial Department of Housing and Urban-Rural Development: Beijing Bangti Conference Co., Ltd., 2119-2122.

[2] Yu, Y.F. (2019) From Traditional Residential Area Planning to Community Living Circle Planning. Urban Planning, 43, 17-22.

[3] Chai, Y.W. and Li, C.J. (2019) Urban Life Circle Planning: From Research to Practice. Urban Planning, 43, 9-16+60.

[4] Zhou, X.X., Wang, P.Z., Yang, F. and Xu, J.G. (2015) Village Layout Planning Based on the Theory of "Living Circle"-Taking Sihong County as an Example. New Normal: Inheritance and Reform-2015 China Urban Planning Annual Conference Proceedings (14 Rural Planning), China Urban Planning Society, Guiyang Municipal People's Government, 1150-1161.

[5] Chai, Y.W., Li, C.J., Xia, W.Q., Wang, J., Zhang, X. and Sun, D.S. (2019) The Delineation Model of Urban Community Life Circle: Taking Qinghe Street in Beijing as an Example. Urban Development Research, 26, 1-8+68.

[6] Zhao, Y.Y., Zhang, B. and Zhou, F. (2018) Research on the Spatial Measurement of Beijing's "15-Minute Community Living Circle” Based on POI. Research World, No. 5, 17-24. 